\title{
Infección periprotésica de rodilla ¿influye la estrategia quirúrgica en el resultado funcional?
}

\author{
Total knee arthroplasty infection, what is the best \\ strategy for better functional outcomes? \\ Crego-Vita D, * Sánchez-Pérez C, ${ }^{\ddagger}$ Espigares-Correa A, ${ }^{\S}$ Aedo-Martín D" \\ Hospital Central de Defensa «Gómez Ulla» de Madrid.
}

RESUMEN. Introducción: La estrategia quirúrgica al enfrentarnos a las infecciones periprotésicas de rodilla sigue siendo controvertida. Los objetivos del tratamiento son la erradicación de la infección, la mejoría del dolor y de la función articular. El manejo quirúrgico incluye el desbridamiento y retención del implante, el recambio protésico en un tiempo o el recambio en dos tiempos. Esta última estrategia quirúrgica se considera el «gold standard», alcanzando unas tasas de curación hasta de $80 \%-100 \%$, aunque poco se ha publicado acerca de los resultados funcionales. Material y métodos: Estudio retrospectivo de 65 pacientes, con infección periprotésica de rodilla. En 20 pacientes se realizó un recambio en un tiempo y en 45 pacientes fueron recambios en dos tiempos. Evaluación pre- y postoperatoriamente con la escala modificada HSS. Todos los pacientes fueron tratados con antibioterapia intravenosa, evaluamos la respuesta analítica y clínica para confirmar la erradicación o no de la infección. Resultados: La infección se resolvió en 39 de 65 pacientes, 12 en el grupo de recambio en un tiempo y 27 para el grupo de dos tiempos. Sin diferencia significativa entre los grupos en relación con curación ni resultado funcional. Sin embargo, hay una diferencia estadísticamente significativa entre aquellos pacientes que recibieron tratamiento antibiótico vía oral antes del diagnóstico y aquéllos que no lo recibieron. Conclusión: La tasa de curación es similar en los tratados con recambio en un tiempo y recambio en dos tiempos. No pudimos demostrar superioridad en los resultados funcionales entre los dos grupos.
ABSTRACT. Introduction: Surgical strategy in dealing with periprosthetic knee infections remains controversial. The goals of treatment are to eradicate infection, improve pain and joint function. Surgical management includes implant debridement and retention, prosthetic replacement in one-time, or two-stage replacement. This latest surgical strategy is considered the «gold standard», reaching healing rates up to $80 \%$ $100 \%$, although little has been published about functional results. Material and methods: Retrospective study of 65 patients with periprosthetic knee infection. In 20 patients a replacement was made in a time and in 45 patients were in two stages. Pre- and post-operative evaluation with the modified HSS scale. All patients were treated with intravenous antibiotherapy, we evaluated the analytical and clinical response to confirm the eradication or not of the infection. Results: The infection was resolved in 39 out of 65 patients, 12 in the replacement group in a time and 27 for the two-stages group. No significant difference between the groups in relation to healing or functional result. However, there is a statistically significant difference between those patients who received oral antibiotic treatment prior to diagnosis and those who did not. Conclusions: The healing rate is similarly treated with replacement in a time and replacement in two times. We were unable to demonstrate superiority in the functional results between the two groups.

\section{Nivel de evidencia: IV}

* Hospital Central de la Defensa «Gómez Ulla», Servicio de Cirugía Ortopédica.

‡ Hospital General Universitario «Gregorio Marañón», Servicio de Cirugía Ortopédica.

${ }^{\S}$ Hospital Rey Juan Carlos, Servicio de Medicina Interna.

" Hospital Universitario del Henares, Servicio de Cirugía Ortopédica.

Dirección para correspondencia:

Diana Crego-Vita, MD PhD

Hospital Central de la Defensa «Gómez Ulla», Servicio de Cirugía Ortopédica.

Glorieta del Ejército SN, CP 28047, Madrid, España.

ORCID ID: 0000-0002-4751-8363

E-mail: crego.diana@gmail.com

Este artículo puede ser consultado en versión completa en: www.medigraphic.com/actaortopedica 
Palabras clave: Infección de prótesis de rodilla, recambio en un tiempo, recambio en dos tiempos, resultados funcionales.

\section{Introducción}

La infección periprotésica (IP) es una de las complicaciones más graves y costosas en cirugía protésica, siendo una de las principales causas de recambio. El diagnóstico de la infección periprotésica no es sencillo y requiere una combinación de marcadores serológicos, técnicas de imagen, anatomía patológica y valoración clínica detenida, ya que no existe ningún test $100 \%$ sensible ni específico. El tratamiento de la IP incluye retirada del implante y sustitución por uno nuevo o bien reimplantación en un segundo tiempo de la prótesis. ${ }^{1}$ En la actualidad, el tratamiento «estándar de oro» se considera el recambio en dos tiempos. ${ }^{2,3,4,5,6}$ No obstante, el recambio en dos tiempos de una artroplastía de rodilla (ATR) se acompaña de morbilidad significativa, hospitalizaciones prolongadas y cambios tisulares severos que pueden producir déficits funcionales. Algunos autores han propuesto realizar recambios en un tiempo en pacientes seleccionados para conseguir una disminución de los costes sanitarios, menor morbilidad y mejor funcionalidad articular. ${ }^{7,8}$

La ATR se ha convertido en una cirugía frecuente, por lo que se espera un aumento en el número de recambios protésicos. En Estados Unidos, por ejemplo, el número anual de procedimientos por recambio protésico relacionados con IP se ha doblado en un período de ocho años. ${ }^{9}$ Una de las causas más frecuentes de revisión protésica es la infección, ya que para controlarla se requiere en muchos casos la retirada del implante. Es importante alcanzar un consenso en relación a la mejor estrategia quirúrgica en estos pacientes: recambio en uno o dos tiempos. Desafortunadamente, el número de trabajos de alta calidad para evaluar los resultados tras revisión de ATR es muy escaso. La mayoría de los estudios se centran en conocer la supervivencia de los implantes, las complicaciones quirúrgicas y la tasa de reinfección; la situación funcional de los pacientes con IP es una información a menudo ausente en la literatura.

El objetivo principal de este trabajo fue evaluar la situación funcional de los pacientes sometidos a recambios en uno o dos tiempos de rodilla. Los objetivos secundarios fueron evaluar la influencia de otros factores sobre la tasa de curación como la edad, índice de masa corporal (IMC), riesgo anestésico (ASA).

Este trabajo presenta una cohorte de pacientes con una ATR infectada que fueron sometidos a recambio protésico. El seguimiento fue mínimo de dos años.

\section{Material y métodos}

Evaluamos retrospectivamente 65 pacientes sometidos a recambio de su ATR. Este trabajo es retrospectivo, no
Keywords: Total knee arthroplasty infection, one stage exchange, two stage exchange, functional outcomes. aleatorizado, realizado en el departamento de Cirugía Ortopédica del Hospital Central de Defensa «Gómez Ulla» de Madrid. Los pacientes fueron tratados entre Febrero de 2011 y Marzo de 2015, recogimos los datos de la historia clínica de los pacientes: edad, sexo, IMC, complicaciones postquirúrgicas, reinfección, número de cirugías previas, tratamientos recibidos previo al recambio, situación funcional mediante escala HSS modificada. Los pacientes fueron contactados por teléfono si algún dato relevante faltaba.

El grupo de recambio en un tiempo corresponde en su mayoría (18 de 20) a pacientes que se recambiaron por dolor sin diagnóstico de infección en el momento de la indicación quirúrgica y en cuyas muestras intraoperatorias microbiológicas creció posteriormente el mismo microorganismo en más de dos muestras. Pasando así de un supuesto recambio aséptico a un recambio por infección, correspondiendo a un tipo IV de Tsukayama. ${ }^{10}$

El grupo de recambio en dos tiempos fueron aquéllos correspondientes al grupo Tsukayama tipo II (infección crónica superior a tres meses desde la implantación de la ATR).

Los criterios de inclusión fueron:

- Pacientes clasificados como Tsukayama II o IV.

- Diagnóstico de infección basado en identificación del microorganismo en líquido articular, presencia de fístula, recuento celular en líquido articular $>2,000 / \mu 1$ leucocitos y $>70 \%$ granulocitos (PMN).

- Consentimiento por parte del paciente para participar en el trabajo.

- Estado de salud satisfactorio con una puntuación de la American Society of Anesthesiologists clasificación (ASA) $\leq$ de 3 .

Los criterios de exclusión fueron:

- Pacientes que no autorizaron participar en el estudio.

- Infección en reimplantación de artroplastía de rodilla.

- Otra infección intercurrente.

- Menos de dos años de seguimiento.

- Defectos óseos severos.

En ambos grupos se retiraron todos los componentes, se tomaron al menos cinco muestras de tejido para estudio microbiológico, en caso de recambio en dos tiempos se tomó una biopsia intraoperatoria de tejido sinovial para recuento de PMN por campo de gran aumento por parte del Servicio de Anatomía Patológica previo a la implantación de componentes. En todos los casos, los sujetos re- 
Tabla 1: Descripción de los grupos sometidos a recambio en uno y dos tiempos,

influencia de diferentes variables y su significación estadística.

\begin{tabular}{|c|c|c|c|}
\hline & Recambio en un tiempo & Recambio en dos tiempos & $\begin{array}{l}\text { Análisis estadístico entre ambos grupos } \\
\text { según la erradicación de la infección (p) }\end{array}$ \\
\hline Edad & 74.8 & 70.8 & 0.098 \\
\hline Sexo & $13 \mathrm{~F} / 7 \mathrm{M}$ & $29 \mathrm{~F} / 16 \mathrm{M}$ & 0.142 \\
\hline ASA & & & 0.541 \\
\hline 1 & 3 & 6 & \\
\hline 2 & 10 & 19 & \\
\hline 3 & 7 & 20 & \\
\hline Etiología & & & 0.784 \\
\hline Osteoartritis & 17 & 37 & \\
\hline Postraumático & 3 & 8 & \\
\hline IMC & 28 & 27 & 0.932 \\
\hline Cirugías previas & 3 & 10 & 0.842 \\
\hline $\begin{array}{l}\text { Uso de antibioterapia vía oral } \\
\text { previa }\end{array}$ & 5 & 31 & 0.002 \\
\hline Preoperatorio Mod HSS & & & 0.866 \\
\hline \multicolumn{4}{|l|}{ Knee score } \\
\hline Excelente & 15 & 26 & \\
\hline Bien & 5 & 15 & \\
\hline Regular & 0 & 4 & \\
\hline Deficiente & 0 & 0 & \\
\hline $\begin{array}{l}\text { Pacientes libres de infección al final } \\
\text { del seguimiento }\end{array}$ & 12 & 27 & \\
\hline \multicolumn{4}{|l|}{$\begin{array}{l}\text { Mod HSS Knee Score al final de } \\
\text { seguimiento }\end{array}$} \\
\hline Excelente & 7 & 11 & \\
\hline Bien & 6 & 20 & \\
\hline Regular & 4 & 10 & \\
\hline Deficiente & 3 & 4 & \\
\hline Exitus letalis & 0 & 3 & \\
\hline
\end{tabular}

cibieron tratamiento antibiótico intravenoso durante dos a cuatro semanas y posteriormente vía oral por cuatro semanas más.

En el grupo diagnosticado de infección tipo IV de Tsukayama con recambio en un tiempo, se implantaron en 12 casos una Advance Revision Stemmed Medial Pivot Knee System (Wright ${ }^{\circledR}$ Arlington TN USA), en los ocho casos restantes se implantó una Legacy constrained condylar knee (LCCK) (Zimmer ${ }^{\circledR}$ Warsaw USA).

En el grupo Tsukayama tipo II con recambio en dos tiempos, se implantó la nueva prótesis entre dos a cuatro semanas después de haber acabado el período de antibioterapia para permitir un período ventana sin antibiótico. Estos pacientes portaron en todos los casos un espaciador de cemento con antibiótico articulado o no para evitar el acortamiento de la extremidad y estabilizar la articulación. El espaciador fue siempre hecho a mano con Copal G+V (Heraeus ${ }^{\circledR}$ Medical GmbH Philipp-Reis-Str. 8/13 61273 Wehrheim Germany) con técnica de mezclado al vacío (Palamix ${ }^{\circledR}$, Heraeus $^{\circledR}$ Medical GmbH Philipp-Reis-Str. 8/13 61273 Wehrheim Germany).

La eficacia del tratamiento antibiótico en el grupo Tsukayama II se controló mediante marcadores serológicos: proteína $\mathrm{C}$ reactiva (PCR) y velocidad de sedimentación globular (VSG). Los valores que se dieron como normales fueron inferiores a $30 \mathrm{~mm} / \mathrm{h}$ y $10 \mathrm{mg} / \mathrm{l}$, respectivamente.
Durante este período, 65 pacientes cumplieron criterios para ser incorporados a este trabajo. Realizamos en 20 casos un recambio en un tiempo con diagnóstico final de Tsukayama II y 45 pacientes con recambio en dos tiempos y diagnóstico de Tsukayama IV. Seis pacientes murieron durante el seguimiento.

En ambos grupos se realizó una valoración funcional preoperatoria y al final del seguimiento mediante la escala modificada HSS Knee Score y de marcadores biológicos incluyendo PCR y VSG. Aunque no haya una definición normalizada de éxito terapéutico, ${ }^{11}$ definimos la curación cuando la infección era controlada sin aparición de fístulas, valores decrecientes de PCR y VSG y ausencia de movilización de los implantes.

\section{Método estadístico}

Se empleó los test de $\chi^{2}$ y t de Student para comparar valores demográficos y comorbilidades entre los grupos. Las diferencias entre los grupos funcionales en el grupo de recambio en un tiempo y dos tiempos para variables discretas (edad, período de seguimiento, PCR, VSG, recuento leucocitario) se compararon empleando el test exacto de Fisher. Un p valor menor de 0.05 se consideró significativo. Todos los análisis estadísticos se realizaron con el programa SPSS software (versión 18; SPSS, Chicago, Illinois). 


\section{Resultados}

\section{Grupo recambio en un tiempo (Tabla 1)}

Veinte pacientes sometidos a recambio de PTR en un tiempo presentaron cultivos positivos en dos o más muestras de tejido, por lo que se consideró que presentaban una infección crónica no identificada y fueron clasificados como Tsukayama IV. Trece mujeres y siete varones con una edad media de 74.8 años \pm 10 -3 (rango 52-89). Cinco pacientes eran diabéticos y un paciente sufría una artritis reumatoide. Tres pacientes fueron clasificados como ASA 1, 10 como ASA 2 y siete como ASA 3. El motivo de la primera sustitución articular fue una gonartrosis primaria en 17 casos y una gonartrosis postraumática en tres casos. El índice de masa corporal (IMC) fue de $28 \pm 6$ (rango 22-34), siete pacientes eran obesos (IMC $>30$ ). Tres pacientes presentaron alguna intervención quirúrgica en esa rodilla previo a la implantación de la PTR (en todos los casos una artroscopía sin material implantado). En cinco casos se inició un tratamiento vía oral con antibiótico por parte de su médico de atención primaria antes de la cirugía de recambio por sospecha de infección articular (rodilla caliente y dolorosa) o infección de partes blandas (celulitis). La puntuación HSS modificada en el momento del diagnóstico de PTR dolorosa era excelente (80-100) en 15 pacientes, buena (70-79) en cinco, deficiente $(60-69)$ en cero y mala $(<60)$ en cero pacientes. Los microorganismos responsables se pueden ver en la Figura 1.

\section{Grupo de recambio en dos tiempos (Tabla 1)}

Cuarenta y cinco pacientes fueron sometidos a un recambio en dos tiempos; 29 mujeres y 16 varones con una edad media de $70.8 \pm 14.4$ (rango 43-92). Cuatro sujetos eran diabéticos y tres presentaban artritis reumatoide. Seis pacientes fueron ASA 1, 19 ASA 2 y 20 ASA 3. La PTR inicial se implantó por gonartrosis primaria en 37 casos y en ocho pacientes se debió a una gonartrosis postraumática. El IMC fue de $27 \pm 5$ (rango 22-40) y 12 sujetos eran obesos. Diez casos presentaron alguna cirugía previa sobre esa rodilla (artroscopía en todos los casos, pero en dos de esos pacientes se realizó una reconstrucción de ligamento cruzado anterior). Treinta y un sujetos fueron tratados con antibioterapia oral antes de la cirugía por presencia de fístulas, aumento de temperatura y calor, y celulitis local por parte de sus médicos responsables. La puntuación HSS modificada en el momento del diagnóstico de la infección fue excelente (80-100) en 26 pacientes, buena (70-79) en 15, deficiente $(60-69)$ en cuatro y mala $(<60)$ en cuatro pacientes. Los microorganismos responsables se muestran en la Figura 1.

Durante el seguimiento murieron seis pacientes, tres muertes no tuvieron relación con la infección, pero las otras tres se debieron a un tromboembolismo pulmonar. La infección se controló en 12 pacientes para el grupo de recambio en un tiempo y en 27 pacientes para el grupo de recambio en dos tiempos. La tasa de éxito a los 24 meses de seguimiento fue de $60 \%$ en los dos grupos. Aquéllos que fueron tratados con antibioterapia oral previo al tratamiento quirúrgico tuvieron dos veces menos posibilidades de erradicar la infección que quienes no recibieron antibioterapia oral $\left(\mathrm{IC}_{95 \%}\right.$ : 1.3-3) $(\mathrm{p}<0.005)$.

Los resultados funcionales de los pacientes pueden observarse en la Tabla 2. No se observaron diferencias estadísticamente significativas entre el grupo de recambio en uno o dos tiempos.

\section{Discusión}

La principal debilidad de este trabajo es el desequilibrio entre ambos grupos: 45 pacientes en el grupo de recambio en dos tiempos y 20 en el grupo de recambio en un tiempo. No obstante, no pudimos demostrar diferencias demográficas entre los dos grupos (sexo, edad, enfermedad inicial que precipitó la implantación de una PTR, nivel funcional previo al recambio, IMC, tasa de complicaciones, reinfección, número de cirugías previas). Sin embargo, sí se evidenció una diferencia estadísticamente significativa al subdividir la muestra entre aquéllos que recibieron un tratamiento antibiótico vía oral antes del recambio y los que no realizaron tratamiento antibiótico previo. En nuestra muestra aquéllos tratados con antibioterapia vía oral antes del recambio, independientemente del motivo (infección respiratoria, celulitis, fístula sobre la prótesis), fueron quienes con más frecuencia presentaron cultivos negativos, lo que impidió la selección del tratamiento antibiótico específico para la infección articular del paciente; de igual manera fueron los que tuvieron menor tasa de erradicación de infección. Esto se puede explicar, puesto que es sabido que para optimizar los resultados microbiológicos es necesario un período ventana libre de antibióticos de al menos dos semanas, ya que las bacterias no son capaces de replicarse tras la presión antibiótica

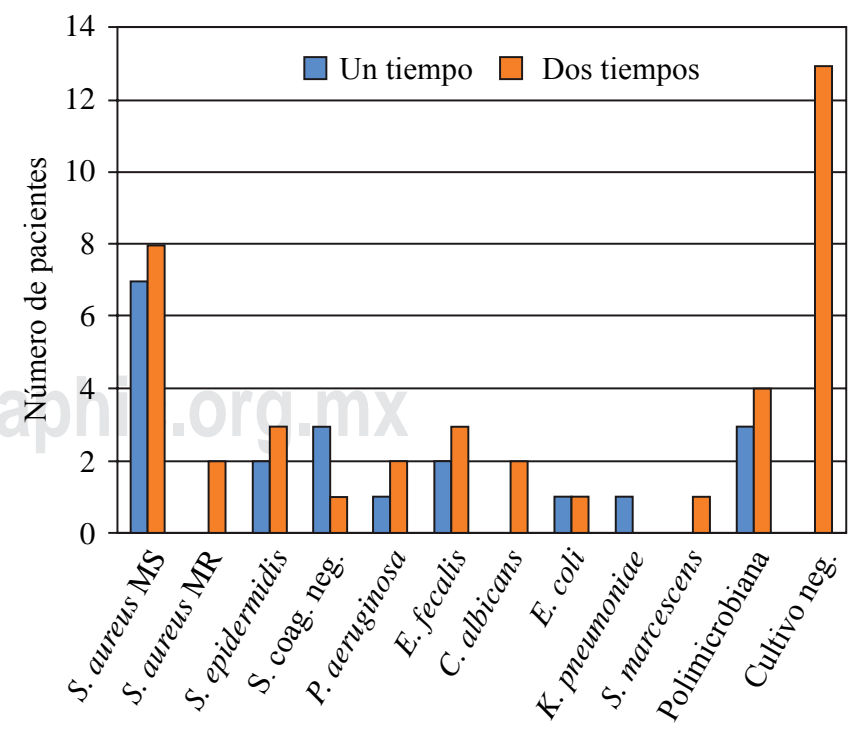

Figura 1: Frecuencia de los microorganismos responsables según el grupo de tratamiento. 


\begin{tabular}{|c|c|c|c|}
\hline & Recambio en un tiempo & Recambio en dos tiempo & Análisis estadístico entre grupos (p) \\
\hline Satisfacción del paciente & & & 0.541 \\
\hline Excelente & 1 & 0 & \\
\hline Buena & 7 & 15 & \\
\hline Regular & 10 & 20 & \\
\hline Baja & 2 & 10 & \\
\hline Dolor (0-10) & & & 0.866 \\
\hline $0-3$ & 15 & 26 & \\
\hline $4-7$ & 5 & 15 & \\
\hline$>7$ & 0 & 4 & \\
\hline Balance articular & & & 0.553 \\
\hline $0-60^{\circ}$ & 7 & 15 & \\
\hline $0-100^{\circ}$ & 6 & 20 & \\
\hline$>100^{\circ}$ & 7 & 10 & \\
\hline
\end{tabular}

a pesar de los medios de enriquecimiento empleados por los especialistas en microbiología. ${ }^{11,12}$ Pensamos que evitar el uso de antibióticos previo a un recambio protésico (mínimo dos semanas) es esencial para la rentabilidad microbiológica y es probable que sea aún más importante de lo que se había pensado hasta ahora, pues parece que influye en la tasa de curación de infección articular.

En nuestro estudio la infección se controló en $60 \%$ de los pacientes. Este dato es ligeramente peor que los publicados hasta la fecha con tasas de fracaso de entre 9 y $34 \%,{ }^{13,14}$ aunque sigue siendo comparable. Asimismo, es probable que nuestros resultados no fueran tan buenos como se esperaba, debido al alto número de pacientes sin identificación de germen responsable durante el tratamiento.

Los criterios para decidir un recambio en un tiempo o en dos tiempos no son comparables con los de otros autores, puesto que en nuestro centro el tratamiento habitual es siempre recambio en dos tiempos. Sólo se realizó recambio en un tiempo porque el motivo de la cirugía no era una infección, sino una ATR dolorosa o ATR movilizada y sólo después se pudo demostrar que el implante estaba infectado. ${ }^{15,16}$ No obstante, los gestos quirúrgicos de limpieza en uno u otro caso son idénticos, tratándose siempre del mismo equipo quirúrgico y realizando de manera sistemática sinovectomía, toma de muestras para microbiología y lavado abundante antes de una nueva implantación. Algunos autores han demostrado que los resultados clínicos entre los dos tipos de técnicas no presentan diferencias tras 18 meses de cirugía, siendo nuestro seguimiento de al menos 24 meses consideramos que es suficiente para poder comparar entre los dos grupos los resultados funcionales. ${ }^{17,18}$

Como otros autores nosotros también defendemos el uso sistemático de biopsia intraoperatoria para recuento de PMN antes de la implantación definitiva en un recambio en dos tiempos. ${ }^{19}$

En nuestra muestra el microorganismo más frecuentemente aislado fue $S$. aureus, siendo este dato coincidente con la bibliografía. ${ }^{20,21}$ Creamos un grupo que englobara los coagulasa negativos excepto para S. epidermidis, ya que existe la tendencia a tratar este microorganismo como patógeno, mientras que el resto de CNS se consideran bacterias saprofíticas. ${ }^{22,23}$ En nuestra muestra el número de pacientes infectados por $S$. aureus y $S$. epidermidis es similar tanto en el grupo de recambio en un tiempo como en el de dos tiempos, lo que hace los resultados comparables.

Al enfrentarnos a recambios protésicos de rodilla por infección no existe consenso en la literatura en relación a la superioridad en cuanto a funcionalidad de una estrategia sobre otra. ${ }^{24} \mathrm{El}$ principal hallazgo de este trabajo es que no detectamos diferencias estadísticamente significativas en los resultados funcionales entre el grupo de recambio en un tiempo y recambio en dos tiempos ni en la puntuación de funcionalidad ni en el rango articular ni en el dolor. Por otro lado los resultados en cuanto al control de la infección son similares en ambos grupos. Trabajos recientes sugieren que los recambios en un tiempo pueden conseguir resultados funcionales superiores siendo una estrategia más costo efectiva: ${ }^{25,26}$ aunque por el momento la evidencia es limitada.

Nuestro trabajo tiene numerosas limitaciones. En primer lugar, el carácter retrospectivo y la obtención de datos de una manera indirecta a través de historias clínicas es criticable. En segundo lugar, los grupos comparados no tienen el mismo tamaño. Además, existen diferentes sesgos que no han sido posibles controlar, ya que el grupo de recambio en un tiempo fueron casos menos severos en cuanto a calidad ósea y ausencia de trastornos tróficos. Los hallazgos de este estudio pueden infraestimar el riesgo relativo de reinfección dado el escaso tiempo de seguimiento ( 24 meses). La principal fortaleza es el empleo de una escala funcional en el seguimiento de los pacientes y una valoración homogénea por el mismo cirujano. ${ }^{27,28}$

La literatura muestra una relativa paridad entre las dos estrategias..$^{29,30}$ Se requieren nuevos estudios en un futuro con mayor control de los posibles sesgos y que permitan una aleatorización entre grupos de tratamientos en uno o dos tiempos. Algunos estudios han demostrado mejores resultados funcionales y mejor percepción subjetiva de los pacientes tras un recambio en un tiempo, ${ }^{25,31}$ pero en número 
son aún escasos. Se requieren más estudios enfocados específicamente a resultados funcionales postquirúrgicos.

A pesar de las limitaciones de nuestro trabajo, encontramos que los dos procedimientos son comparables en términos de resultados funcionales y tasas de reinfección, otros autores encontraron resultados similares. ${ }^{29,32,33}$ Aunque la supuesta superioridad hasta la fecha de los recambios en dos tiempos hayan podido desanimar a la comunidad científica a diseñar trabajos comparativos entre ambas estrategias, la falta de evidencia que existe hoy en día debería estimularnos a desarrollar trabajos aleatorizados comparativos que nos permitan conocer mejor lo que podemos esperar de cada técnica.

\section{Conclusión}

El recambio en un tiempo de una PTR parece igual de efectivo que el recambio en dos tiempos en términos de erradicación de infección con resultados funcionales similares. Por otra parte, es esencial evitar los tratamientos antibióticos por vía oral previo al tratamiento quirúrgico de una infección periprotésica si queremos aspirar a tener los mejores resultados posibles en relación al control de la infección.

\section{Bibliografía}

1. Parvizi J, Gerkhe T, Chen AF. Proceedings of the international consensus on periprosthetic joint infection. Bone Joint J. 2013; 95B(11): 1450-2.

2. Teeny SM, Dorr L, Murata G. Treatment of infected total knee arthroplasty. J Arthroplasty. 1990; 5: 35-9.

3. Wilson MG, Kelley K. Thornhill TS. Infection as a complication of total knee replacement arthroplasty. J Bone Joint Surg Am. 1990; 72: 878-83.

4. Hirakawa K, Stulberg BN, Wilde AH. Results of 2 stage reimplantation for infected total knee arthroplasty. J Arthroplasty. 1998; 13: 22-8.

5. Haleem AA, Berry DJ, Hanssen AD. Mid term to long term follow up of two stage reimplantation for infected total knee arthroplasty. Clin Orthop Relat Res. 2004; 428: 35-9.

6. Hofman AA, Goldberg T, Tanner AM, Treatment of infected total knee arthroplasty using an articulated spacer 2-12 year experience. Clin Orthop Relat Res. 2005: 430: 125-31.

7. Tibrewal S, Malagelada F, Jeyaseelan L, Posch F, Scott G. Single stage revision for the infected total knee replacement: results from a single centre. Bone Joint J. 2014; 96B(6): 759-64.

8. Singer J, Merz A, Frommelt L, Fink B. High rate of infection control with one stage revision of septic knee prosthesis excluding MRSA and MRSE. Clin Ortop Relat Res. 2012; 470(5): 1461-71.

9. Kurtz M, Lau EC, Ong KL, Adler EM, Kolisek FR, Manley MT. Economic burden of periprosthetic joint infection in the United States. J Arthroplasty. 2012; 27(8 Suppl): 61-5.e1.

10. Tsukuyama DT, Estrada R, Gustilo RB. Infection after total hip arthroplasty: a study of the treatment of one hundred and six infection. J Bone Joint Surg Am. 1996; 78(4):512-23.

11. Parvizi J, Fassihi SC, Enayatollahi MA. Diagnosis of periprosthetic joint infection following hip and knee arthroplasty. Orthop Clin North America. 2016; 47(3): 505-15.

12. Malekzadeh D, Osmon DR, Lahr BD, et al. Prior use of antimicrobial therapy is a risk factor for culture negative prosthetic joint infection. Clin Orthop Relat Res. 2010; 468(8): 2039-45.
13. Cobo J, Del Pozo JL. Prosthetic joint infection: diagnosis and management. Expert Rev Anti Ther. 2011; 9(9): 787-802.

14. Jämsen E, Stogiannidis I, Malmivaara A, Pajamäki J, Puolakka T, Konttinen YT. Outcome of prosthesis exchange for infected knee arthroplastya: the effect of treatment approach. Acta Orthop. 2009; 80(1): 67-77.

15. Masters JP, Smith NA, Foguet P, Reed M, Parsons H, Sprowson AP. A systematic review of the evidence for single stage and two stage revision of infected knee replacement. BMC Musculoskelet Disord. 2013; 14(1): 222.

16. Callaghan JJ, Katz RP, Johnston RC. One stage revision surgery of the infected hip: a minimum 10 year follow up study. Clin Orthop Relat Res. 1999; 369: 139-43.

17. Bachmeier CJ, March LM, Cross MJ, et al. A comparison of outcomes in osteoarthritis patients undergoing total hip and knee replacement surgery. Osteoarthritis Cartilage. 2001; 9: 137-46.

18. Fortin PR, Penrod JR, Clarke AE, St-Pierre Y, Joseph L, Bélisle P, et al. Timing of total joint replacement affects clinical outcomes among patients with osteoarthritis of the hip or knee. Arthritis Rheum. 2002; 46: 3327-46.

19. Raut W, Siney PD, Wroblewski BM. One stage revision of infected total hip replacement with discharging sinuses. J Bone Joint Surg Br. 1994; 76: 721-4.

20. Javier Ariza, Gorane Euba y Óscar Murillo. Infecciones relacionadas con las prótesis articulares. Enferm Infecc Microbiol Clin. 2008; 26(6): 380-90

21. Zhang M, Yan CH, Chan PK, Yuen F, Chiu KY. Polyethilene insert exchange is crucial in debridement for acute periprosthetic infections following total knee arthroplasty. J Knee Surg. 2016; 30: 36-41.

22. McCann M T, Gilmore B F, Gorman S P. Staphylococcus epidermidis device-related infections: pathogenesis and clinical management. $J$ Pharm Pharmacol. 2008; 60(12): 1551-71.

23. Kloos W E, Bannerman T L. Update on clinical significance of coagulase-negative staphylococci. Clin Microbiol Rev. 1994; 7: 117-40.

24. Banit DM, Kaufer H, Hartford JM. Intraoperative frozen section analysis in revision total joint arthroplasty. Clin Orthop Relat Res. 2002; 401: 230-8.

25. Nagra NS, Hamilton TW, Ganatra S, Murray DW, Pandit H. Onestage versus two-stage exchange arthroplasty for infected total knee arthroplasty: a systematic review. Knee Surg Sports Traumatol Arthrosc. 2016; 24(10): 3106-14.

26. Haddad FS, Sukeik M, Alazzawi S. Is single-stage revision according to a strict protocol effective in treatment of chronic knee arthroplasty infections? Clin Ortop Relat Res. 2014; 473: 8-14.

27. Modified Hospital for Special Surgery Knee Scoring System. Journal of Orthopaedic Trauma. 2006; 20(8): S100-S101.

28. Insall JN, Dorr LD, Scott RD, Scott WN. Rationale of the knee society clinical rating system. Clin Orthop Relat Res. 1989; (248): 13-4.

29. Dzaja I, Howard J, Somerville L, Lanting B. Functional outcomes of acutely infected knee arthroplasty: a comparison of different surgical treatment options. J Can Chir. 2015; 58: 402-7.

30. Leonard HA, Liddle AD, Burke O, Murray DW, Pandit H. Single or two-stage revision for infected total hip arthroplasty? a systematic review of the literature. Clin Orthop and Relat Res. 2014; 472: 1036-42.

31. Oussedik SI, Dobb MB, Haddad FS. Outcomes of revision total hip replacement for infection after grading according to a standard protocol. J Bone Joint Surg Br. 2010; 92: 1222-6.

32. Bourne RB, Chesworth BM, Davis AM, Mahomed NN, Charron KD. Patient satisfaction after total knee arthroplasty: who is satisfied and who is not? Clin Orthop Relat Res. 2010; 468: 57-63.

33. Moore AJ, Bloom AW, Whitehouse MR, Gooberman-Hill R. Managing uncertainty - a qualitative study of surgeons' decision making for one-stage and two-stage revision surgery for prosthetic hip joint infection. BMC Musculoskelet Disord. 2017; 18(1): 154. 